Check for updates

Cite this: RSC Adv., 2017, 7, 21982

\title{
Gold nanoparticles as analytical tools for the quantification of small quantities of triazine derivatives anchored on graphene in water dispersions $\dagger$
}

\begin{abstract}
Verónica León, ${ }^{a}$ Gregorio Castañeda, ${ }^{\mathrm{b}}$ María Antonia Herrero ${ }^{\mathrm{a}}$ and Ester Vázquez (DD *a
The development of new methodologies for the preparation of aqueous graphene dispersions is contributing to fostering their biological applications. However, there is still a need for analytical methods to fully characterise the different graphene materials and to identify the presence of other components in the medium in order to establish structure-behaviour correlations. We report here a fast, simple and sensitive method to detect ppm levels of melamine anchored on graphene in aqueous graphene dispersions. Gold nanoparticles can be directly cross-linked with melamine and the resulting aggregation produces a change in colour that can be readily detected in the presence of graphene. Visible changes can be observed with the naked eye and determined by UV-Vis spectroscopy. MALDITOF was used to confirm the results obtained with the colorimetric methodology. Elemental analysis results were employed as the standard measurements.
\end{abstract}

Received 25th February 2017

Accepted 7th April 2017

DOI: $10.1039 / \mathrm{c} 7 \mathrm{ra0} 3358 \mathrm{~h}$

rsc.li/rsc-advances

\section{Introduction}

Graphene is a versatile material with amazing properties such as high thermal conductivity, high charge carrier mobility, large specific surface area and excellent mechanical properties. ${ }^{1}$ All of these features, combined with the wide range of possible modifications, make graphene and graphene derivatives valuable resources in a vast number of areas.

In recent years, biological and biomedical applications of graphene have emerged and several encouraging results have been reported in fields such as drug delivery, enhanced cell differentiation and growth, biological imaging and biosensing. ${ }^{2}$ However, considerable challenges remain, such as the need for systematic studies on the toxicity and environmental impact of graphene and graphene derivatives based on chemical and physical properties, which require adequate classification standards for graphene derivatives in order to identify structure-activity relationships. ${ }^{3}$

One of the bottlenecks in the use of graphene and graphene derivatives in biological and biomedical studies has been the solubility of these materials in water and culture media.

${ }^{a}$ Departamento de Química Orgánica, Inorgánica y Bioquímica, Facultad de Ciencias y Tecnologías Quimicas-IRICA, Universidad de Castilla-La Mancha, Campus Universitario, 13071 Ciudad Real, Spain.E-mail: Ester.vazquez@uclm.es

${ }^{b}$ Departamento de Quimica Analitica y Tecnología de Alimentos, Facultad de Ciencias y Tecnologías Quimicas-IRICA, Universidad de Castilla-La Mancha, Campus Universitario, 13071 Ciudad Real, Spain

$\dagger$ Electronic supplementary information (ESI) available. See DOI: 10.1039/c7ra02358h
Graphene oxide is soluble in water and has been extensively used for biomedical applications, ${ }^{4}$ but aqueous dispersions of non-oxidized graphene materials are difficult to obtain. Different approaches to achieve this goal include the exfoliation of graphite in aqueous media in the presence of polymers, anionic surfactants, ${ }^{5,6}$ pyrene derivatives ${ }^{7}$ or even proteins. ${ }^{8,9}$ However, the amounts of additive necessary to maintain pristine graphene in solution are, in general, too high and they leave only small proportions of the graphene surface accessible for further interactions with biological targets. Moreover, in order to use graphene materials in biological applications, it is very important to identify new methods for their characterisation and standardization in order to define better which type of materials and preparation approaches are safe and suitable for these studies.

Our research group has developed an easy methodology for the preparation of graphene suspensions in water and culture media. The exfoliation of graphite sheets is achieved by interactions with triazine derivatives in a ball milling process under solvent free conditions. ${ }^{10,11}$ The behaviour of triazine derivatives as exfoliating agents has been studied by ab initio calculations. ${ }^{11}$ Moreover, the interaction of water molecules with the graphene surface in the presence of a molecule of melamine $(2,4,6-$ triamino-1,3,5-triazine) has been analysed. Repeated washing of the graphene water dispersions with hot water remove the majority of melamine but residual amounts were always present on the graphene surface. Without this negligible amount of melamine, graphene was no longer soluble in water. $A b$ initio calculation showed that this small functional molecule act as an 
anchor on the graphene surface and at the same time can form $\mathrm{H}$-bonds with water molecules. ${ }^{12}$ The stability of these graphene dispersions in water and culture media has been studied ${ }^{\mathbf{1 3}}$ and this has allowed biomedical investigations into the preparation of hydrogels for drug delivery purposes, ${ }^{\mathbf{1 4}}$ the preparation of nerve cell interfaces or the study of the synaptic functions in culture brain cells ${ }^{15}$ and in ecotoxicological investigations. ${ }^{16}$

However, some concerns could arise from the use of triazine derivatives for the preparation of graphene dispersions for use in biomedical studies. Triazine derivatives have been used as herbicides, for the production of resins, and as additives in paints, plastic and papers. ${ }^{17,18}$ As result of these applications, triazine derivatives have been found in the environment and, in large quantities, they are responsible for a variety of toxic effects. ${ }^{\mathbf{1 9 , 2 0}}$ Melamine is undoubtedly the most famous of these derivatives and notorious cases of toxicity have been reported. Indeed, the ingestion of melamine at levels above the safety limit (2.5 ppm in the United States; $1 \mathrm{ppm}$ for infant formula in China) can induce renal failure and even death in infants. ${ }^{21}$ As a consequence, several methods have been developed for the rapid and selective detection of these compounds. ${ }^{22,23}$ Nevertheless, the identification of residual amounts of molecules on the surface of graphene is not an easy task. The characterisation of graphene materials relies on expensive and sophisticated equipment such as Atomic Force Microscopy, (AFM), Scanning Tunneling Microscopy (STM) and Transmission Electron Microscopy (TEM), among others, and there is a need for accurate methodologies able to routinely and promptly detect non-covalently absorbed substances on these new materials even if they are in solution. Many of the analytical methodologies employed for the quantitative determination of triazine derivatives are not applicable to graphene systems because they are based on chromatographic techniques that require special sample preparation or need fluorescent sensors in which the presence of graphene may interfere by signal quenching. In order to avoid these problems, we selected a colorimetric method based on gold nanoparticles and this avoids timeconsuming treatments.

Gold nanoparticles (AuNPs) with diameters in the range 10$50 \mathrm{~nm}$ in water absorb green light, which corresponds to a strong absorption band at $520 \mathrm{~nm}$ in the visible spectrum. These solutions are red in colour. A target analyte that directly triggers AuNP aggregation can be detected by the colour change of the AuNP solution, which can be observed by the naked eye. Triazine derivatives contain multiple sites that allow strong interactions with the surface of AuNPs and these play the role of a molecular linker to efficiently crosslink AuNPs, which can be directly observed by the naked eye or monitored by ultraviolet visible (UV-vis) absorbance spectroscopy. ${ }^{24,25}$ However, considering graphene dispersions as analytes and metal nanoparticles as analytical tools is not a straightforward task. ${ }^{26}$ Standardised methods, reported in the literature for the detection of melamine, resulted to be not suitable for the detection of melamine adsorbed on graphene (see below). Graphene materials are commonly used as supports to stabilized metal nanoparticles, and it is important to determine if this fact could compromise the analysis of the triazine derivatives.
Besides providing a fast, reliable and straightforward analytical methodology for the detection and quantification of ppm levels of melamine anchored on graphene in aqueous dispersions, the work presented here can serve as the basis for the analysis of alternative molecules that may be present in graphene dispersions or non-covalently anchored on graphene surfaces (Fig. 1).

\section{Experimental}

\section{Materials}

Melamine was purchased from Sigma-Aldrich and was used as received without further purification. Graphite was purchased from Bay Carbon, Inc. (SP-1 graphite powder, http:// www.baycarbon.com) and was used without purification. Gold(III) chloride hydrate and trisodium citrate dihydrate were purchased from Sigma-Aldrich.

\section{Synthesis of graphene dispersions in water}

Graphite was first exfoliated through interactions with melamine (2,4,6-triamino-1,3,5-triazine) by ball milling under solvent free conditions. ${ }^{10} 7.5 \mathrm{mg}$ of graphite and $22.5 \mathrm{mg}(0.18$ $\mathrm{mmol}$ ) of melamine were ball-milled at $100 \mathrm{rpm}$ for 30 minutes in an air atmosphere. Once the graphene had been exfoliated, the melamine was removed by filtration with hot water. Graphene samples were then redispersed in fresh water to obtain stable dispersions. Elemental analysis was used as the standard method for the quantification of melamine and therefore for the validation of our analytical methods.

\section{Synthesis of AuNPs}

AuNPs were prepared by reducing of gold(III) chloride trihydrate with trisodium citrate. ${ }^{27}$ The resulting citrate-stabilized AuNPs were wine-red owing to their strong surface plasmon resonance at $520 \mathrm{~nm}$. In a typical procedure, $25 \mathrm{~mL}$ of trisodium citrate (38.8 mM) was added to a boiling solution of gold(III) chloride trihydrate (500 mL, $0.5 \mathrm{mM}$ ) and the solution was stirred for 15 minutes until a wine-red suspension was obtained. The concentration of the AuNPs in suspension was determined by UV-Vis spectroscopy, as reported in the literature, ${ }^{28}$ and a $5.2 \mathrm{nM}$ AuNP suspension was obtained.

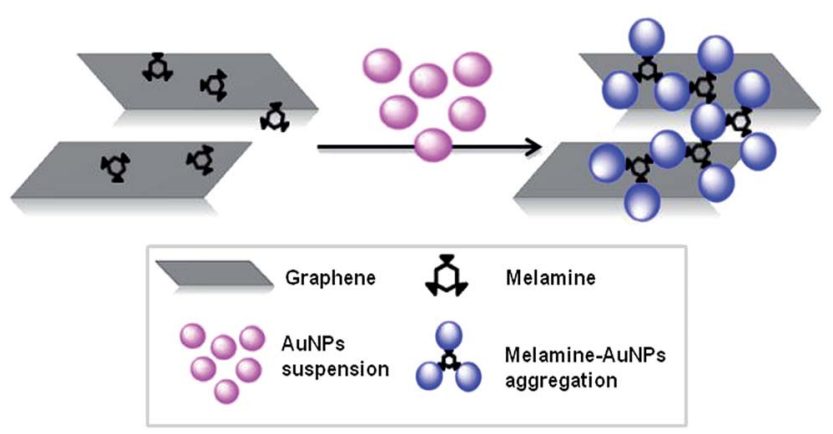

Fig. 1 Interaction of melamine in graphene samples with AuNPs. 


\section{Detection of melamine}

In a typical procedure, $1 \mathrm{~mL}$ of $5.2 \mathrm{nM}$ AuNP suspension was added to $2 \mathrm{~mL}$ of graphene dispersions with different concentrations of melamine. The colour change was observed by the naked eye and recorded by UV-vis spectrophotometry. The aggregation of AuNPs with melamine was determined by measuring the absorbance spectra one minute after the addition.

\section{Characterisation}

The milling treatments were carried out in a Retsch PM100 Planetary Mill. Elemental analysis was performed on a LECO CHNS-932 analyser (Model no. 601-800-500). UV-vis-NIR spectra were recorded in $1 \mathrm{~cm}$ quartz cuvettes on a Cary $5000 \mathrm{UV}$-vis-NIR spectrophotometer. Mass spectra were measured with an Autoflex II time-of-flight (TOF)/TOF Bruker spectrometer (Bremen, Germany). The melamine/graphene dispersions were analysed in positive reflector mode in the $m / z$ range $50-300$ with an optimised fixed laser. 2000 shots per spectrum were accumulated randomly from 3 spot positions for each example. For TEM analyses the dispersions were placed on a copper grid (3.00 mm, $200 \mathrm{mesh}$, coated with carbon film) and the sample was investigated by High-Resolution Transmission Electron Microscopy (HRTEM) JOEL 2100.

\section{Results and discussion}

\section{Colorimetric method for detection of melamine}

Graphene dispersions prepared by exfoliation of graphite through interaction with melamine contain traces of melamine, which could hinder the use of this material in biological studies. Therefore, in order to scale-up the process easily it is important to quantify, in a simple fast and effective way, the final level of melamine that remains in the graphene dispersions.

Initial efforts to reproduce published reports ${ }^{24}$ on the detection of melamine using gold nanoparticles were unsuccessful. In fact, we found out that the response on aggregation of $\mathrm{Au}$ nanoparticles in free melamine samples and melamine adsorbed on graphene is different, giving erroneous results (see ESI $\dagger$ ). For example, the addition of small amounts of melamine, dispersed in water, to a control graphene-melamine dispersion prepared by ball milling treatments, gave a wrong calibration curve. For this reason, a graphene dispersion in water with 3.5 $\mu \mathrm{g} \mathrm{mL}^{-1}$ of melamine (determined by elemental analysis) was used as the starting solution. This sample was employed to prepare new graphene dispersions $\left(0.42 \mu \mathrm{g} \mathrm{mL} \mathrm{m}^{-1}\right.$ to $2.9 \mu \mathrm{g}$ $\mathrm{mL}^{-1}$ ) containing melamine concentrations in the range 3.5$0.5 \mu \mathrm{g} \mathrm{mL} \mathrm{m}^{-1}$ by dilution. A $5.2 \mathrm{nM}$ solution of nanoparticles in water was added to the graphene-melamine dispersions and the colour of the AuNPs progressively changed from wine red to blue as the melamine concentration increased.

The corresponding colorimetric effect was evaluated by considering the ratio between absorbance at $640 \mathrm{~nm}$ and at $520 \mathrm{~nm}$ (A640/A520) for each dilution. However, the results showed poor linearity, which suggests that a high melamine concentration $\left(>2.5 \mu \mathrm{g} \mathrm{mL}^{-1}\right)$ leads to the rapid precipitation of AuNPs during the mixing of melamine with AuNPs, signifying that kinetic aggregation cannot be accurately characterised by UV-Vis spectroscopy at these concentrations. ${ }^{24}$ We therefore chose lower melamine concentrations $\left(0.2 \mu \mathrm{g} \mathrm{mL} \mathrm{mL}^{-1}\right.$ to $2.0 \mu \mathrm{g}$ $\mathrm{mL}^{-1}$ ) for the preparation of the calibration curve.

The absorption bands at $520 \mathrm{~nm}$ and $640 \mathrm{~nm}$, which correspond to the original gold colloid and AuNPs-melamine aggregates, respectively, are comparable with the bands observed in the presence of graphene. ${ }^{24}$ Only an increase of the baseline signal was observed due to the absorption of graphene. ${ }^{29}$ Therefore, the minimum of each spectrum obtained at $800 \mathrm{~nm}$ was used as a baseline. This finding suggests that AuNPs do not attach to graphene in the absence of the melamine, due to the strong electrostatic repulsions between the two dispersions. This situation is consistent with the results of a study in which the AuNPs were not incorporated onto the graphene oxide (GO) surface if the GO was not coated with an organic molecule, which allows the capture of the AuNPs from the solution. ${ }^{30,31}$

A graphene dispersion in water, with $2.7 \mu \mathrm{g} \mathrm{mL}{ }^{-1}$ of melamine (determined by elemental analysis), was employed to prepare new graphene dispersions $\left(20 \mu \mathrm{g} \mathrm{mL}^{-1}\right.$ to $\left.150 \mu \mathrm{g} \mathrm{mL}^{-1}\right)$ containing a melamine concentration in the range 2-0.2 $\mu \mathrm{g}$ $\mathrm{mL}^{-1}$ by dilution. In each case the melamine concentrations were calculated by elemental analysis.

Direct exposure of nanoparticles to graphene dispersions led to a progressive colour change in the AuNPs from wine red to blue (Fig. 2) as the melamine concentration increased in the graphene dispersion. The most clear colour changes were observed at a melamine concentration of $\leq 1.1 \mu \mathrm{g} \mathrm{mL} \mathrm{m}^{-1}$.

The aggregation of AuNPs with melamine was monitored by UV-Vis spectroscopy (Fig. 3a). The addition of melamine at different concentrations led to a gradual decrease in the original absorbance of AuNPs at $520 \mathrm{~nm}$, while a new absorbance at $640 \mathrm{~nm}$, which is characteristic of the aggregates, increased. These results suggest that traces of melamine in graphene dispersions can be directly detected by aggregation with the AuNPs. The corresponding colorimetric effect was evaluated by comparing the ratio between the absorbance at $640 \mathrm{~nm}$ and the absorbance at $520 \mathrm{~nm}$ (A640/A520) for each dilution of the graphene dispersion (Fig. $3 \mathrm{~b}$ ).

\section{Validation of the proposed colorimetric method}

The linearity of the assay was evaluated using different graphene dispersion samples containing melamine at concentrations in the range from 0.2 to $2 \mu \mathrm{g} \mathrm{mL}^{-1}$. The calibration curve was determined from duplicate absorbance ratio measurements (A640/A520) using a least squares linear regression analysis. The

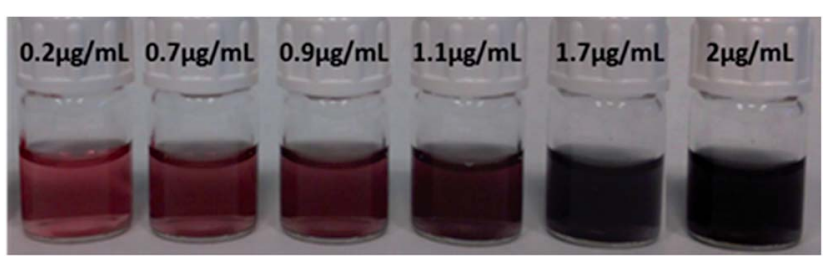

Fig. 2 Visual colour change of AuNPs in graphene dispersions, which indicated the concentration of melamine. 

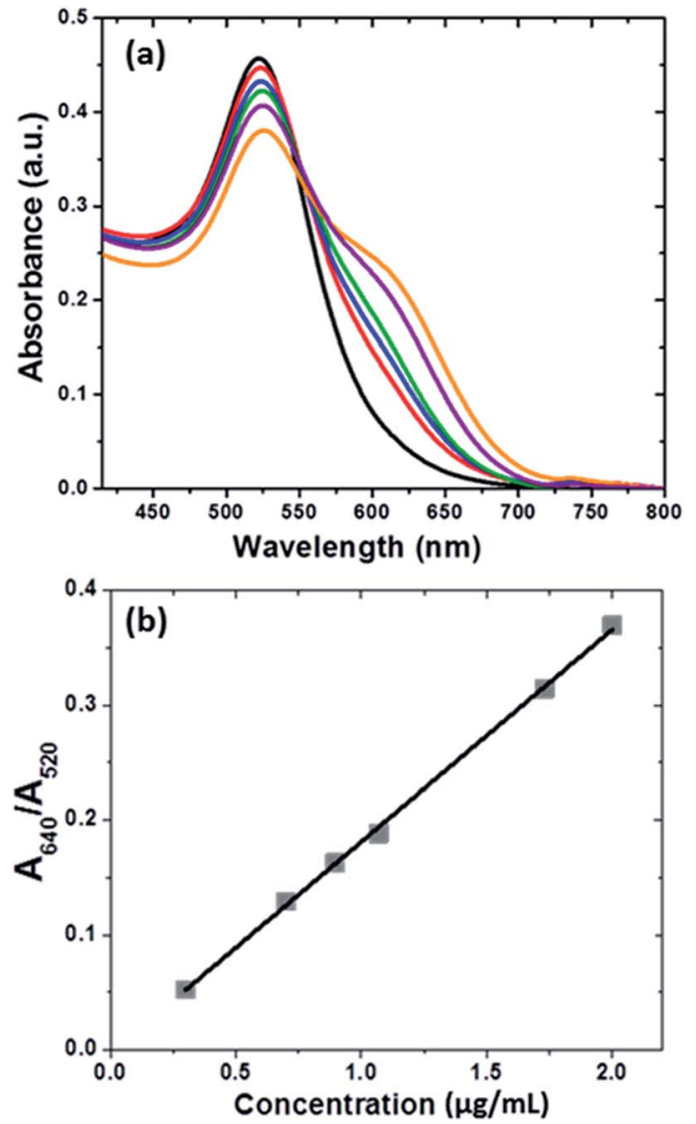

Fig. 3 (a) The evolution of UV-Vis absorbance spectra of AuNPs with graphene dispersions at different concentrations of melamine: 0.3 (black), 0.7 (pink), 0.9 (blue), 1.1 (green), 1.7 (purple) and $2 \mu \mathrm{g} \mathrm{mL}^{-1}$ (brown). (b) Ratio A640/A520 versus melamine concentration of graphene dispersion.

satisfactory coefficient of determination confirmed that the melamine response was linear over the concentration range studied (Table 1). The measurements confirmed that AuNPs provide a reliable assay for the rapid detection of melamine in graphene dispersions.

The LOD was obtained as the concentration of melamine corresponding to an A640/A520 signal three times higher than the baseline noise level. The LOQ was calculated as three times the LOD. In this way, LOD and LOQ were established as $0.04 \mu \mathrm{g}$ $\mathrm{mL}^{-1}$ and $0.15 \mu \mathrm{g} \mathrm{mL} \mathrm{mL}^{-1}$, respectively. The LOQ was

Table 1 Results from the statistical analysis

\begin{tabular}{lll}
\hline & $\begin{array}{l}\text { Spectrophotometric method } \\
\text { (A640/A520) }\end{array}$ & MS method \\
\hline Intercept & $-3.14 \times 10^{-3} \pm 9.59 \times 10^{-3}$ & $-6.01 \pm 4.45$ \\
Slope & $1.85 \times 10^{-1} \pm 7.62 \times 10^{-3}$ & $26.64 \pm 4.10$ \\
$R^{2}$ & 0.9991 & 0.9921 \\
Linear range $\left(\mu \mathrm{g} \mathrm{mL}^{-1}\right)$ & $0.2-2.0$ & $0.2-2.0$ \\
LOD $\left.(\mu \mathrm{g} \mathrm{mL})^{-1}\right)^{a}$ & 0.04 & 0.20 \\
LOQ $(\mu \mathrm{g} \mathrm{mL})^{a}$ & 0.15 & 0.66 \\
${ }^{a}$ IUPAC. & &
\end{tabular}

subsequently validated experimentally by analysis of samples at this concentration level.

The precision of the proposed method was investigated in terms of repeatability and intermediate precision, for the absorbance ratio considered, and the results are expressed as relative standard deviation (RSD). The repeatability was studied by analysing sequentially six graphene dispersion samples containing melamine at a concentration level of $0.9 \mu \mathrm{g} \mathrm{mL} \mathrm{m}^{-1}$. The RSD of A640/A520 absorbance rate values was $13.6 \%$ and the average concentration was $0.86 \mu \mathrm{g} \mathrm{mL}^{-1}$. Regarding intermediate precision, the same operation was repeated on different days. RSD values of less than $13.6 \%$ (13.1\%) were obtained and an average concentration of $0.86 \mu \mathrm{g} \mathrm{mL}{ }^{-1}$ was found. The two sets of data were compared with the aim of detecting random errors by applying the Snedecor F-test on these RSD values. Significant differences were not found in any case at a confidence level of $95 \%$ and five degrees of freedom. Comparison of the average concentration on consecutive days by a $t$-test did not show any significant differences at a confidence level of $95 \%$.

In order to test the accuracy of the proposed method, addition/ recovery assays were performed. In different volumetric flasks, several graphene dispersion samples containing melamine concentrations between $0.2 \mu \mathrm{g} \mathrm{mL} \mathrm{m}^{-1}$ and $2.0 \mu \mathrm{g} \mathrm{mL} \mathrm{mL}^{-1}$ were prepared. These samples were then analysed in duplicate according to the proposed method. Recoveries were calculated against the external standards included in the sequence of samples. The results for analysed samples are shown in Table 2. It can be seen that recoveries ranging between $92 \%$ and $110.7 \%$ were obtained.

Further direct evidence for melamine/AuNPs aggregates was characterised by Transmission Electron Microscopy (TEM) (Fig. 4). TEM images show that AuNPs are well-dispersed with an average size of $20 \mathrm{~nm}$ (Fig. 4a). However, the melamine in the graphene dispersion stimulated aggregation of AuNPs (Fig. 4b).

In order to confirm the accuracy of the method, dispersions with different concentrations of melamine were also characterised by matrix-assisted laser desorption/ionisation (MALDI). Persike et $a l . .^{32}$ described a quick and easy method for the analysis of melamine and its derivatives in standard solutions and in milk powder using matrix-assisted laser desorption/ionisation timeof-flight mass spectrometry (MALDI-TOF-MS). A dried-droplet method was used in which the MALDI matrix was sinapinic acid.

However, it has been found in several studies that graphene can act as a matrix for the analysis of low molecular weight

Table 2 Recoveries of melamine in graphene dispersion samples

\begin{tabular}{lllr}
\hline Sample & $\begin{array}{l}\text { Concentration of } \\
\text { melamine }^{a}\left(\mu \mathrm{g} \mathrm{mL}{ }^{-1}\right)\end{array}$ & $\begin{array}{l}\text { Melamine concentration } \\
\text { found }\left(\mu \mathrm{gL}^{-1}\right)\end{array}$ & $\begin{array}{l}\text { Recovery } \\
(\%)\end{array}$ \\
\hline 1 & 0.37 & 0.34 & 91.9 \\
2 & 0.93 & 1.03 & 110.7 \\
3 & 1.36 & 1.43 & 105.1 \\
4 & 0.87 & 0.87 & 101.1 \\
5 & 0.79 & 0.80 & 101.2 \\
\multicolumn{2}{l}{${ }^{a}$ Elemental analysis. } & &
\end{tabular}



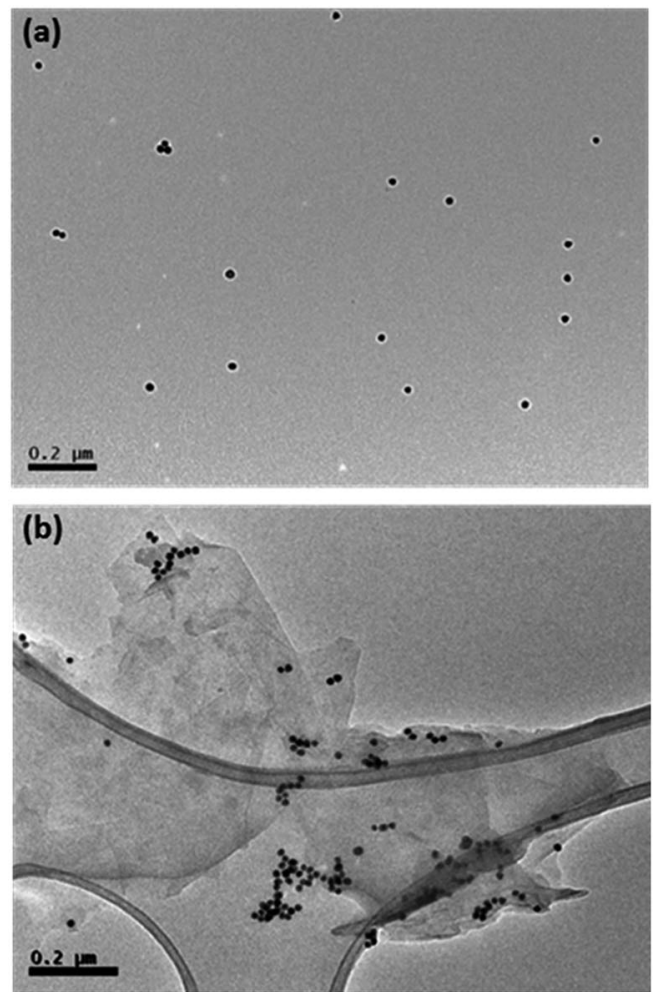

Fig. 4 TEM images of AuNPs (a) and graphene dispersion with the addition of AuNPs (b).

compounds by MALDI-TOF-MS. ${ }^{33}$ Thus, in this study the analysis was carried out without additional matrix.

In a typical procedure, $10 \mu \mathrm{L}$ of each dispersion were placed into three wells of the target plate and these samples were completely dried at room temperature before being inserted into a vacuum-based mass spectrometer for analysis. The mass spectrum of the graphene sample showed one characteristic peak at $\mathrm{m} / z$ 128.264, which corresponds to the melamine that is present in the graphene dispersion. The mass spectrum of each dilution was acquired three times and the average signal-

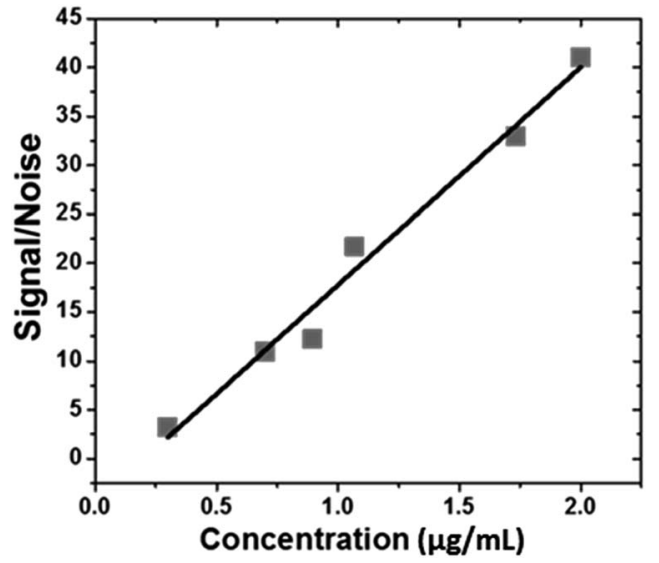

Fig. 5 Signal-to-noise ratio versus melamine concentration of graphene dispersion by MALDI-TOF-MS method.

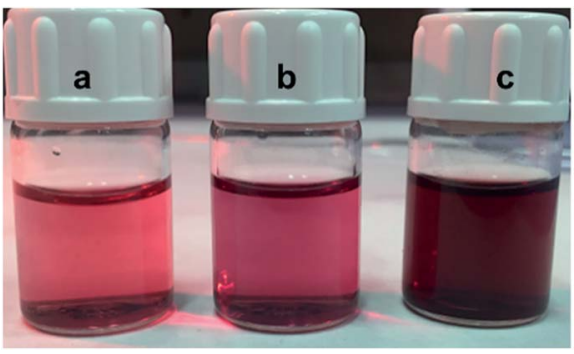

Fig. 6 Visual colour change of AuNPs in graphene dispersions, (a) 2.2 $\mu \mathrm{g} \mathrm{mL}^{-1}$ (b) $6.8 \mu \mathrm{g} \mathrm{mL}^{-1}$ to (c) $11.2 \mu \mathrm{g} \mathrm{mL}^{-1}$ of 6-(4-nitrophenyl)-2,4diamine-1,3,5-triazine anchored on the graphene surface.

to-noise $(\mathrm{S} / \mathrm{N})$ ratio was plotted against concentration of melamine in the graphene dispersion, with Beer- Lambert behaviour observed (Fig. 5). The different samples used to validate the colorimetric method were also evaluated by MALDI-TOF-MS and the efficacy of the colorimetric method described to quantify the melamine in the presence of graphene was confirmed.

Finally, further studies are being carried out in our laboratory with the aim of extending this analytical approach to other triazine derivatives. ${ }^{11}$ Preliminary results show that the direct exposure of gold nanoparticles to aqueous graphene dispersions that contain small amounts of 6-(4-nitrophenyl)-2,4-diamine1,3,5-triazine on the surface, produces colour changes that can be correlated with increasing concentrations of the triazine derivative. (Fig. 6).

\section{Conclusions}

We have developed an easy methodology to provide the on-site and real-time detection of melamine as a non-covalent anchor on graphene in aqueous dispersions. The method has high sensitivity, rapid detection, simplicity and quantitative accuracy. The method is useful to confirm when possible traces of melamine are below safety limits in order to perform biological studies. Moreover, the methodology paves the way for the analysis of small amounts of other molecules anchored on graphene in water dispersions.

\section{Acknowledgements}

This work was supported, in part, by AEI/FEDER, UE and the Spanish Ministerio de Economía y Competitividad (project CTQ2016-78793-P and project CTQ2014-53600-R) and EU Graphene-based disruptive technologies, Flagship project (no. 696656). The Maldi-Tof-Tof spectrometer was acquired by FEDER-JCCM funding, UNCM05-23-061.

\section{Notes and references}

1 C. Si, Z. Suna and F. Liu, Nanoscale, 2016, 8, 3207-3217.

2 W. Yang, K. R. Ratinac, S. P. Ringer, P. Thordarson, J. J. Gooding and F. Braet, Angew. Chem., Int. Ed., 2010, 49(12), 2114-2138. 
3 P. Wick, A. E. Louw-gaume, M. Kucki, H. F. Krug, K. Kostarelos, B. Fadeel, K. A. Dawson, A. Salvati, E. Vazquez, L. Ballerini, M. Tretiach, F. Benfenati, E. Flahaut, L. Gauthier, M. Prato and A. Bianco, Angew. Chem., Int. Ed., 2014, 53, 7714-7718.

4 E. Abbasi, A. Akbarzadeh, M. Kouhi and M. Milani, Artif. Cells, Nanomed., Biotechnol., 2014, 1-7.

5 M. Lotya, P. J. King, U. Khan, S. De and J. N. Coleman, ACS Nano, 2010, 4, 3155-3162.

6 M. Lotya, Y. Hernandez, P. J. King, R. J. Smith, V. Nicolosi, L. S. Karlsson, F. M. Blighe, S. De, Z. Wang, I. T. McGovern, G. S. Duesberg and J. N. Coleman, J. Am. Chem. Soc., 2009, 131(10), 3611-3620.

7 F. Irin, M. J. Hansen, R. Bari, D. Parviz, S. D. Metzler, S. K. Bhattacharia and M. J. Green, J. Colloid Interface Sci., 2015, 446, 282-289.

8 S. Ahadian, M. Estili, V. J. Surya, J. Ramón-Azcón, X. Liang, H. Shiku, M. Ramalingam, T. Matsue, Y. Sakka, H. Bae, K. Nakajima, Y. Kawazoe and A. Khademhossein, Nanoscale, 2015, 7, 6436-6443.

9 A. Pattammattel and C. V. Kumar, Adv. Funct. Mater., 2015, 25, 7088-7098.

10 V. León, M. Quintana, M. A. Herrero, J. L. G. Fierro, A. de la Hoz, M. Prato and E. Vázquez, Chem. Commun., 2011, 47(39), 10936-10938.

11 V. León, A. M. Rodriguez, P. Prieto, M. Prato and E. Vázquez, ACS Nano, 2014, 8(1), 563-571.

12 A. M. Rodriguez, A. B. Muñoz-García, O. Crescenzi, E. Vázquez and M. Prato, Phys. Chem. Chem. Phys., 2016, 18, 22203-222209.

13 V. León, J. M. Gonzalez-Dominguez, J. L. G. Fierro, M. Prato and E. Vázquez, Nanoscale, 2016, 8, 14548-14555.

14 A. Servant, V. Leon, D. Jasim, L. Methven, P. Limousin, E. V. Fernandez-Pacheco, M. Prato and K. Kostarelos, Adv. Healthcare Mater., 2014, 1-10.

15 A. Fabbro, D. Scaini, V. Leon, E. Vázquez, G. Cellot, G. Privitera, L. Lombardi, F. Torrisi, F. Tomarchio, S. Bosi, A. C. Ferrari, L. Ballerini and M. Prato, ACS Nano, 2015, 10(1), 615-623.

16 M. Kucki, P. Rupper, C. Sarrieu, M. Melucci, E. Treossi, A. Schwarz, V. León, A. Kraegeloh, E. Flahaut, E. Vázquez, V. Palermo and P. Wick, Nanoscale, 2016, 8, 8749-8760.
17 V. D. M. Finete, M. M. Gouvea, F. F. D. Marques and A. D. P. Netto, Talanta, 2014, 123, 128-134.

18 W. C. Andersen, S. B. Turnipseed, C. M. Karbiwnyk, S. B. Clark, M. R. Madson, C. M. Gieseker, R. A. Miller, N. G. Rummel and R. Reimschuessel, J. Agric. Food Chem., 2008, 56, 4340-4347.

19 Federal Insecticide, Fungicide, and R. A. (FIFRA). U.S. EPA (U.S. Environmental Protection Agency) (2004)http:// www.epa.gov/opp00001/regulating/fifra, accessed 2 Jan 2005.

20 T. Gebel, S. Kevekordes, K. Pav, R. Edenharder and H. Dunkelberg, Arch. Toxicol., 1997, 71, 193-197.

21 C. M.-E. Gossner, J. Schlundt, P. Ben Embarek, S. Hird, D. LoFo-Wong, J. J. O. Beltran, K. N. Teoh and A. Tritscher, Environ. Health Perspect., 2009, 117(12), 1803-1808.

22 Y. Li, J. Xu and C. Sun, RSC Adv., 2015, 5, 1125-1147.

23 P. Kaleeswaran, T. Nandhini and K. Pitchumani, New J. Chem., 2016, 40, 3869-3874.

24 H. Chi, B. Liu, G. Guan, Z. Zhang and M.-Y. Han, Analyst, 2010, 135(5), 1070-1075.

25 R. K. Bera and C. R. Raj, Analyst, 2011, 136(8), 1644-1648.

26 Á. I. López-Lorente and M. Valcárcel, Trends Anal. Chem., 2016, 75, 1-9.

27 K. C. Grabar, R. G. Freeman, M. B. Hommer and M. J. Natan, Anal. Chem., 1995, 67(4), 735-743.

28 W. Haiss, N. T. K. Thanh, J. Aveyard and D. G. Fernig, Anal. Chem., 2007, 79(11), 4215-4221.

29 M. Lotya, P. J. King, U. Khan, S. De and J. N. Coleman, ACS Nano, 2010, 4(6), 3155-3162.

30 J. Liu, S. Fu, B. Yuan, Y. Li and Z. Deng, J. Am. Chem. Soc., 2010, 132, 7279-7281.

31 F. Bei, X. Hou, S. L. Y. Chang, G. P. Simon and D. Li, Chem.Eur. J., 2011, 17, 5958-5964.

32 A. Arnold, T. N. Arrey, M. Karas and M. Persike, Rapid Commun. Mass Spectrom., 2011, 25(19), 2844-2850.

33 X. Dong, J. Cheng, J. Li, Y. Wang, W. Haiss, N. T. K. Thanh, J. Aveyard, D. G. Fernig, K. C. Grabar, R. G. Freeman, M. B. Hommer, M. J. Natan, M. M. T. Blaze, A. Akhmetov, B. Aydin, P. D. Edirisinghe, G. Uygur and L. Hanley, Anal. Chem., 2010, 82, 6208-6214. 